\title{
EDITORIAL
}

\section{Recycled Polyethylene Terephthalate Waste for Different Application Solutions}

\begin{tabular}{l|l|l}
\hline Virginija & Function Materials Centre, Kaunas University of Technology & Kaunas, Lithuania \\
\cline { 2 - 3 } Jankauskaitè & virginija.jankauskaite@ktu.lt \\
\hline
\end{tabular}

For the last 150 years plastics have contributed to the development and progress of society. There is no activity where plastics do not play a key role (from clothing to shelter, from transportation to communication, from entertainment to health care). Plastics, because of its attractive properties, such as lightweight, high strength and ease of processing meet a large share of the materials. There are six main plastics which arise in municipal solid waste - high density polyethylene, low density polyethylene, polyethylene terephthalate, polystyrene, polypropylene and polyvinyl chloride.

Poly(ethylene terephthalate) (PET) is thermoplastic polyester widely used in diverse application as textile fibres, films, bottles and other moulded products. Depending on PET processing and thermal history, it may exist both as an amorphous (transparent) and as a semi-crystalline (opaque and white) material. PET monomer can be synthesized by the esterification reaction between terephthalic acid and ethylene glycol with water as a by-product, or the transesterification reaction between ethylene glycol and dimethyl terephthalate with methanol. One of the main reasons for the PET widespread use is their possibility of producing a number of different grades over a broad range of molecular weights in a single multiproduct polymerization plant.

Over the recent years, the amount of different products from PET has been particularly increasing, resulting in the greater quantities of household polymeric waste. In Lithuania companies produce about 10 thousand tonnes of PET containers per year. The huge amounts of PET products (bottles, spinning, packaging films) cause serious environmental pollution. Commonly, PET content reaches about $12 \%$ in municipal plastic waste. In fact, the separation of PET bottles from municipal waste represents one of the most successful examples of polymer recycling.

Among all plastics PET has received particular attention in terms of post-consumer recycling due to the relatively large availability of PET bottles from special collection scheme. PET occupies the top of the list of polymers to be recycled due to its easy recycling by different ways and receive variable products. Numerous ways of recycling disposable beverage PET bottles are available, including methods of chemical recycling or physical recycling by re-melting. In Lithuania more than $20 \mathrm{com}$ panies recycle plastic waste. The joint stock companies such as "Polivektris", "Plastiksè", "Ecoservice", etc. provide PET waste recycling.

Plastic recycling is very important for the least two main reasons: firstly, to reduce the increasing volumes of plastic waste and secondly, to generate value-added materials from low cost sources by converting them into valuable materials. PET is fully recyclable and is used for manufacturing new products in many indus- 
trial areas (fabrics, high-quality carpets, packaging for detergents, cosmetics, foils, car spare parts, pillow fillings for allergic persons).

The physically recycled PET blends with polycarbonate, polyolefinic elastomer, etc., prepared by co-rotating twin-extruder by melt blending, are considered to be interesting combinations for obtaining new high-performance polymeric materials without synthesizing fully new polymers especially when the properties of two or more polymers are synergistically combined. It is also one of the most important routes of polymer recycling because it makes the sorting step much simpler during recycling operation.

Unfortunately, PET with most polymers is immiscible, and their blends show poor mechanical properties and unstable morphology. These problems can be overcome by using various kinds of compatibilizers. PET blend with maleic anhydride modified styrene butadiene rubber show homogeneous dispersions, high adhesion between dispersed and matrix phase and improved mechanical properties. It can be applied to manufacture micro-fibrillar reinforced composites comprising of an isotropic matrix from a lower melting polymer, reinforced by micro-fibrils of a higher melting polymer. LDPE (matrix) and recycled PET (reinforced material) from bottles were melt-blended and extruded, followed by continuous drawing, palletizing and injection moulding. Extruded blend was isotropic, but becomes highly oriented after drawing, being converted into a polymer-polymer composite upon injection moulding at temperatures below the melting temperature of PET.

The possibilities for the use of recycled PET in powder form as filler in the polyvinyl chloride, polychloroprene or other matrix also exist. To enhance the degree of interaction and compatibility, the surface of powdered PET can be modified by plasma.

The products of PET chemical recycling are widely used in the production of unsaturated polyesters, polyester moulding compounds, topcoats of reinforced plastics, varnishes, binders for glass-fabrics, pre-pregs, polymer plasters, mortars, and concrete materials with mineral fillers as well as fibres, polyoles for polyurethane elastomers, foams, and polyurethanes of low flammability.
PET can be chemically processed by total depolymerization into monomers or partial depolymerization into oligomers and other products. Different recycling processes for chemical reprocessing or chemolysis of PET waste are available and most of them consist of esterifying the polyester with an excess of reactants such as water (hydrolysis), alcohols (alcoholysis), amines (aminolysis), ammonia (ammonolysis) and glycols (glycolysis). The focus mainly on new trends of glycolysis reactions, as this process is more advantageous from the economical point of view than other kinds of chemolysis is made. The companies such as "Eastman Kodak", "Goodyear", "Du Pont", "Zimmer" use mainly glycolysis for PET recycling. Glycolysis reaction is the molecular degradation of PET polymer by glycols, in the presence of trans-esterification catalysts, mainly metal acetates, where ester linkages are broken and replaced with hydroxyl terminals. PET waste can be depolymerized by glycolysis to obtain oligomeric diols and polyols, or glycolyzed into its monomeric units, bis(2-hydroxyeth$\mathrm{yl}$ ) terephthalate or dimethyl terephthalate. Bis(2-hydroxyethyl) terephthalate can be used as a substrate to create PET and other polymers.

In the PET recycling procedure obtained oligoesters can be further reacted with aliphatic diacids to form polyester polyols that are used as a starting material in the polyurethane industry. Rigid polyurethane foams prepared from the product of PET degradation with triethanolamine can be applied as insulation materials. The depolymerization products of PET by ethylene glycol, diethylene glycol, polyethylene glycol-200 and glycerin were transesterified with the glycolyzed products of soy bean oil and glycerin. The urethane varnish oils finally obtained showed a broad spectrum of physical properties that are matching and, in some instances, are better than those of commercial grades. As a new motivation, PET waste was depolymerized with a number of special diols and used for the synthesis of novel block copolymers. Oligoester diols coming from depolymerizing PET waste were introduced as reducing agents in these redox polymerization reactions, where the resulting polymers were suggested to have chain ends of the corresponding reducing agent moiety and, hence, affected the final physical properties of the resulting copolymers. The process is commercially important, first 
because it converts a waste material to a value-added product, and second, because it gives polyester polyols that have terephthalate-repeating units, which overcomes the problem of sublimation arising from direct use of terephthalic acid as a starting material in the polyol synthesis.

Unsaturated polyester resins based on recycled PET have been used as a matrix for high performance fiber-reinforced polymer composites that replace conventional structural materials like steel and wood in a variety of engineering applications. These high-performance composites offer excellent chemical resistance, weather-ability, high strength to weight ratio and ease of fabrication. Unsaturated polyester resin synthesized from PET waste is also applied in making polymer concretes and polymer mortars.

PET waste can be processed to give radiation curable oligomers. With increasing regulatory pressure to reduce solvent emissions, many coating processes have adopted radiation curing, using either ultraviolet or electron beam irradiation. Such coating processes are intrinsically green, since they do not use any vol- atile organic solvents that would give rise to air pollution. Specific application areas include printing inks, and metal and wood coatings. Acrylates are the most commonly used monomers and irradiation initiated free radical processes lead to curing. These formulations typically include an acrylate terminated urethane oligomer, a multi-functional acrylate for cross-linking and mono-acrylate reactive diluents. PET waste can be converted to either acrylate or methacrylate-terminated PET oligomers by a two-stage process, first glycolysis with diethylene glycol followed by reaction with either acryloyl chloride or methacryloyl chloride. The oligomers were viscous oils that could be blended with commercial radiation-curable oligomers, cast into films and cured by UV irradiation. These results raise the possibility of converting waste PET into high value-added radiation-curable oligomers for coatings applications.

Thus, PET recycling provides useful possibilities. Physically recycled PET is widely used in the blends with other polymers, while chemically recycled PET can be introduced as starting ingredients for synthesis of many other polymers. 